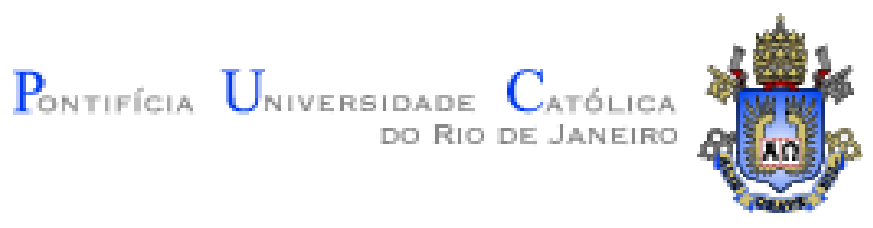

Felipe Duarte Cardozo de Pina

The homology of some isospectral manifolds

Thesis presented to the Postgraduate Program in Mathematics of the Departamento de Matemática, PUC-Rio as partial fulfillment of the requirements for the degree of Mestre em Matemática Pura.

Advisor : $\quad$ Prof. Carlos Tomei

Co-Advisor: Prof. Nicolau Corção Saldanha 


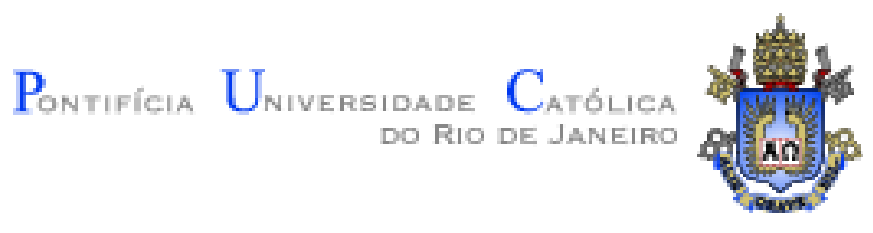

Felipe Duarte Cardozo de Pina

\title{
The homology of some isospectral manifolds
}

Thesis presented to the Postgraduate Program in Mathematics, of the Departamento de Matemática do Centro Técnico Científico da PUC-Rio, as partial fulfillment of the requirements for the degree of Mestre.

\author{
Prof. Carlos Tomei \\ Advisor \\ Departamento de Matemática - PUC-Rio
}

Prof. Nicolau Corção Saldanha

Co-Advisor

Departamento de Matemática - PUC-Rio

Prof. Ricardo Soares Leite Departamento de Matemática - UFES

Prof. Paul Alexander Schweitzer, S. J. Departamento de Matemática - PUC-Rio

Prof. Henrique Bursztyn Instituto de Matemática Pura e Aplicada - IMPA

Prof. José Eugenio Leal Coordinator of the Centro Técnico Científico da PUC-Rio

Rio de Janeiro - September 08, 2008 
All rights reserved

\section{Felipe Duarte Cardozo de Pina}

Graduated in Pure Mathematics from Pontifícia Universidade Católica do Rio de Janeiro in 2004.

Bibliographic data

Pina, Felipe Duarte Cardozo de
The homology of some isospectral manifolds / Felipe
Duarte Cardozo de Pina ; advisor: Carlos Tomei; co-advisor:
Nicolau Corção Saldanha. - 2008.
50 f. : il. ; $30 \mathrm{~cm}$
Dissertação (Mestrado em matemática)-Pontifícia Uni-
versidade Católica do Rio de Janeiro, Rio de Janeiro, 2008.
Inclui bibliografia
1. Matemática - Teses. 2. Variedades isospectrais. 3.
Fluxos de Toda. 4. Teoria de Morse. 5. Homologia. 6.
Tightness. 7. Tautness. I. Tomei, Carlos. II. Saldanha,
Nicolau Corção. III. Pontifícia Universidade Católica do Rio
de Janeiro. Departamento de Matemática. IV. Título.

CDD: 510 


\section{Acknowledgments}

To my advisors, professors Carlos Tomei and Nicolau Saldanha, for teaching me, in such a delightful manner, a great deal of beautiful mathematics and for giving me a glimpse of the world of mathematical research.

To my dear colleagues, for lending me their ears and sharing their ideas.

To my beatiful wife Gabriella for her love and support.

To my dear friend Lilia for helping me through the most difficult moments.

To CAPES and PUC-Rio, for their financial support, without which this work would not have been realized.

To my friend and $\mathrm{LT}_{\mathrm{E} X}$ wizard Guilherme Frederico for his priceless hints.

To the staff of the Mathematics Departament for their efficient help. 


\section{Abstract}

Pina, Felipe Duarte Cardozo de; Tomei, Carlos; Saldanha, Nicolau Corção. The homology of some isospectral manifolds. Rio de Janeiro, 2008. 50p. Dissertação de Mestrado — Departamento de Matemática, Pontifícia Universidade Católica do Rio de Janeiro.

For $\Lambda$ a real, diagonal matrix of simple spectrum, we consider $\mathcal{O}_{\Lambda}$, the isospectral manifold of real, symmetric matrices conjugate to $\Lambda$, and $\mathcal{T}_{\Lambda}$, the isospectral manifold of tridiagonal matrices in $\mathcal{O}_{\Lambda}$. We compute the homologies of both manifolds, combining techniques of Morse theory and integrable systems. As a consequence, we show that the immersion of $\mathcal{O}_{\Lambda}$ in the vector space of real symmetric matrices is tight and taut, a fact with implications in numerical spectral theory.

\section{Keywords}

Isospectral manifolds. Toda flows. Morse theory. Homology. Tightness. Tautness. 


\section{Resumo}

Pina, Felipe Duarte Cardozo de; Tomei, Carlos; Saldanha, Nicolau Corção. Homologia de variedades isoespectrais. Rio de Janeiro, 2008. 50p. Dissertação de Mestrado — Departamento de Matemática, Pontifícia Universidade Católica do Rio de Janeiro.

Para $\Lambda$ uma matriz diagonal real de espectro simples, consideramse $\mathcal{O}_{\Lambda}$, a variedade de matrizes reais, simétricas conjugadas a $\Lambda$, e $\mathcal{T}_{\Lambda}$, a variedade das matrizes tridiagonais em $\mathcal{O}_{\Lambda}$. Calcula-se as homologias das duas variedades, combinando técnicas de teoria de Morse e sistemas integráveis. Como conseqüência, mostra-se que a imersão de $\mathcal{O}_{\Lambda}$ no espaço vetorial de matrizes reais simétricas é tight e taut, o que tem implicações em teoria espectral numérica.

\section{Palavras-chave}

Variedades isospectrais. Fluxos de Toda. Teoria de Morse. Homologia. Tightness. Tautness. 


\section{Resumo Expandido}

Seja $\Lambda=\operatorname{diag}\left(\lambda_{1}, \ldots, \lambda_{n}\right)$ uma matriz real, $n \times n$, diagonal e de espectro simples, ou seja, com $n$ autovalores distintos $\lambda_{1}<\ldots<\lambda_{n}$. Consideramos duas variedades, o conjunto $\mathcal{O}_{\Lambda}$ de matrizes reais $n \times n$, simétricas conjugadas a $\Lambda$ e seu subconjunto $\mathcal{T}_{\Lambda}$ das matrizes tridiagonais em $\mathcal{O}_{\Lambda}$. Seja $\mathcal{S}_{n}$ o espaço vetorial real das matrizes simétricas de ordem $n$.

O fato que $\mathcal{O}_{\Lambda}$ é uma variedade compacta orientável naturalmente mergulhada em $\mathcal{S}_{n}$ é essencialmente uma aplicação do teorema da função implícita. Por razões mais elaboradas, as mesmas propriedades valem para $\mathcal{T}_{\Lambda}$. Nosso principal objetivo é calcular as homologias $H_{*}\left(\mathcal{T}_{\Lambda} ; \mathbb{Z}\right)$ e $H_{*}\left(\mathcal{O}_{\Lambda} ; \mathbb{Z} / 2 \mathbb{Z}\right)$. Isto é suficiente para obtermos alguma informação geométrica interessante sobre o mergulho natural $\mathcal{O}_{\Lambda} \hookrightarrow \mathcal{S}_{n}$ — este é tight e taut, definidos abaixo um resultado com conseqüências inesperadas em teoria espectral de matrizes. Este não é o caso para $\mathcal{T}_{\Lambda}$, como mostraremos, mas ainda assim apresentaremos algumas funções de Morse perfeitas nesta variedade.

O ingrediente básico é a função altura

$$
h(S)=\operatorname{tr} D S,
$$

onde $D=\operatorname{diag}\left(d_{1}, \ldots, d_{n}\right)$ com números distintos $d_{k}$. Funções altura são de Morse em $\mathcal{O}_{\Lambda}$ e em $\mathcal{T}_{\Lambda}$, sugerindo o cálculo das homologias através do procedimento tradicional de teoria de Morse (10).

Resumidamente, calculamos seqüencialmente a homologia dos subconjuntos $\left.M_{c}=h^{-1}(-\infty, c]\right)$ para valores crescentes de $c$. O tipo topológico de $M_{c}$ muda nos valores críticos de $h$ de uma maneira controlada. Um cálculo simples mostra que os pontos críticos de $h$ são as matrizes diagonais $\Lambda^{\pi}=\operatorname{diag}\left(\lambda_{\pi(1)}, \ldots, \lambda_{\pi(n)}\right)$, onde $\pi \in S_{n}$ é uma permutação. Em $\mathcal{T}_{\Lambda}, \Lambda^{\pi}$ é um ponto crítico de índice $i$ se e somente se a permutação $\pi$ tem $i$ descidas (descents), i.e., $d(\pi)=i$ (para terminologia e notação relacionadas a permutações, vide Apêndice 8.5). $\operatorname{Em} \mathcal{O}_{\Lambda}$, o índice do mesmo ponto crítico é o número de inversões (inversions) $i(\pi)$.

A dificuldade reside no entendimento dos connecting homomorphisms $\partial: H_{k+1}\left(M_{c+\epsilon}, M_{c-\epsilon} ; A\right) \rightarrow H_{k}\left(M_{c-\epsilon} ; A\right)$ associados à colagem de uma nova célula na transição por um valor crítico $c=h\left(\Lambda^{\pi}\right)$. Ocorre que todos os connecting homomorphisms são triviais, tanto para $\mathcal{T}_{\Lambda}$ quanto para $\mathcal{O}_{\Lambda}$, para uma escolha adequada de coeficientes. Portanto, a homologia essencialmente conta os pontos críticos de um dado índice.

Para verificar a trivialidade destas aplicações, usamos o Fluxo de Toda,

$$
\dot{S}=\left[S, \Pi_{a} S\right]
$$


uma equação diferencial especial que mantém ambas as variedades invariantes. Aqui, $\Pi_{a} S$ é a matriz real anti-simétrica cuja parte triangular inferior é igual à de $S$. O argumento é consideravelmente simplificado pelo fato de que nas variedades existem estruturas riemannianas para as quais o gradiente da função altura é o campo $\left[S, \Pi_{a} S\right]$ de Toda.

De fato, um gradiente genérico de uma função altura $h$ pode ser difícil de acompanhar, mas as variedades estáveis e instáveis do fluxo de Toda podem ser calculadas explicitamente, e é dessa forma que se tornam úteis no estudo dos connecting homomorphisms.

Para $\mathcal{T}_{\Lambda}$, o gerador da homologia relativa $H_{k+1}\left(M_{c+\epsilon}, M_{c-\epsilon} ; A\right)$ em um ponto crítico $\Lambda^{\pi}$ é homotópico a um ciclo $\gamma$ contido na variedade estável $W_{s}\left(\Lambda^{\pi}\right)$. Este ciclo, como será visto, é trivializado pelo fecho de $W_{s}\left(\Lambda^{\pi}\right)$, que vem a ser uma variedade orientável. A conseqüência é que $H_{*}\left(\mathcal{T}_{\Lambda} ; \mathbb{Z}\right)$ é gerado de forma livre por tais fechos.

O argumento para $\mathcal{O}_{\Lambda}$ precisa ser refinado. Neste caso, o fecho de $W_{s}\left(\Lambda^{\pi}\right)$ não é uma variedade, mas ainda nos dá uma trivialização para o gerador de $H_{k+1}\left(M_{c+\epsilon}, M_{c-\epsilon} ; A\right)$ quando as contas são realizadas em $\mathbb{Z} / 2 \mathbb{Z}$.

Em (12), foi mostrado que o conjunto $\mathcal{T}_{\Lambda}$ tem $\mathbb{R}^{n-1}$ por seu recobrimento universal. Pouco depois, Fried (5) calculou seu anel de cohomologia usando técnicas relacionadas às utilizadas neste texto. A variedade $\mathcal{O}_{\Lambda}$ é, em um certo sentido, mais simples, sendo uma órbita adjunta para uma ação de grupo apropriada (para matrizes complexas), à qual técnicas de grupos de Lie podem ser aplicadas. De fato, usando idéias de Bott, Faybusovich e Kocherlakota calcularam $H_{*}\left(\mathcal{O}_{\Lambda}\right)$ em (4) e (6), respectivamente.

Pelos resultados acima, a função altura $h$ definida em cada variedade é perfeita: para matrizes diagonais $D$ de espectro simples, $h$ é uma função de Morse cujo número de pontos críticos de índice $k$ é igual ao $k$-ésimo número de Betti. Pelas desigualdades de Morse, este é o número mínimo de pontos críticos para uma função de Morse em uma variedade compacta.

Uma pequena amplificação deste fato tem uma interpretação geométrica interessante: o mergulho $\iota: \mathcal{O}_{\Lambda} \hookrightarrow \mathcal{S}_{n}$ é tight e taut, no sentido que, para qualquer hiperplano $\mathcal{P} \subset \mathcal{S}_{n}$ (resp. ponto $S \in \mathcal{S}_{n}$ ), a função altura $h_{\mathcal{P}}: \mathcal{O}_{\Lambda} \rightarrow \mathbb{R}$ que calcula a distância (com sinal) de um ponto $p \in \mathcal{O}_{\Lambda}$ a $\mathcal{P}$ (resp. $\ell_{S}: \mathcal{O}_{\Lambda} \rightarrow \mathbb{R}$, o quadrado da distância de $p \in \mathcal{O}_{\Lambda}$ a $S$ ), se Morse, é perfeita (24). Este não é o caso para o mergulho análogo de $\mathcal{T}_{\Lambda}$ no espaço de matrizes reais, tridiagonais, simétricas: mostramos um contra-exemplo para matrizes $3 \times 3(25)$.

Em geral, tightness implica a two-piece property (TPP): um mergulho $M \subset \mathbb{R}^{n}$ satisfaz a TPP se, para todo hiperplano $\mathcal{P} \subset \mathbb{R}^{n}$, o complemento 
$M \backslash \mathcal{P}$ contém no máximo duas componentes conexas. Como exposto em (2), uma formulação equivalente da TPP para uma variedade compacta imersa $M$ é que toda função altura de Morse admite um único mínimo local e um único máximo local. Em outras palavras, para tais funções, um extremo local é necessariamente global. Concluímos o texto com algumas aplicações naturais dessas idéias a problemas em teoria espectral numérica. 


\section{Contents}

$\begin{array}{llr}1 & \text { Introduction } & 11\end{array}$

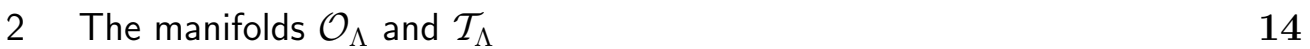

$\begin{array}{lll}2.1 & \text { Triangular coordinates } & 14\end{array}$

3 The Toda Flow $\quad 18$

$\begin{array}{lll}3.1 \text { Construction } & 18\end{array}$

$\begin{array}{ll}3.2 & \text { The Toda equations } \\ & 19\end{array}$

4 Height functions $\quad 24$

4.1 The Toda vector field and $\nabla h_{D} \quad 24$

5 Toda asymptotics $\quad 26$

5.1 Stable and unstable manifolds in $\mathcal{T}_{\Lambda} \quad 26$

5.2 Stable and unstable manifolds in $\mathcal{O}_{\Lambda} \quad 28$

$6 \quad$ Computing homologies $\quad 31$

$7 \quad$ Tightness and Tautness 35

$\begin{array}{ll}\text { Bibliography } & 38\end{array}$

8 Appendix $\quad 39$

$\begin{array}{lll}8.1 & \text { Basic notation } & 39\end{array}$

$\begin{array}{lll}8.2 & \text { Eigen-Smoothness } & 39\end{array}$

8.3 The manifolds $O(n)$ and $S O(n) \quad 42$

8.4 $Q R, L U$ and Bruhat decompositions 45

$\begin{array}{lll}8.5 & \text { Permutations and inversions } & 46\end{array}$

$\begin{array}{lll}8.6 & \text { Stable and unstable manifolds } & 47\end{array}$

8.7 Transition maps 48

$\begin{array}{lll}8.8 & \text { Orientability of } \mathcal{T}_{\Lambda} & 49\end{array}$ 